\title{
Einstein's Equivalence Principle. An Explicit Statement and Its Derivation from Special Relativistic Presymmetry*
}

\author{
Y. Avishai ${ }^{\star \star}$ \\ Argonne National Laboratory, Argonne, Ill., USA

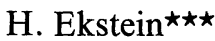 \\ Argonne National Laboratory, Argonne, Ill., USA and \\ Centre Universitaire Marseille-Luminy, Marseille, France \\ Received April 15, 1973; in revised form February 6, 1974
}

\begin{abstract}
We propose a statement of the observable implications of the equivalence principle that is precise and general rather than allusive and illustrative. This statement is derived from previously formulated principles of special-relativistic presymmetry. The substantial identity of our result with Einstein's curved-space formulation is exhibited.
\end{abstract}

\section{Introduction}

This paper challenges the traditional view that the equivalence principle conflicts with special relativity and requires a nonlinear space-time. The principle of special relativity was stated in an operationally explicit manner, including external fields, in previous papers $[1-4]$. The assumptions, slightly augmented, are used to derive a result which, we believe, is a more explicit form of Einstein's equivalence principle.

The idea that many results of Einstein's theory can be obtained within the framework of Minkowski space $M$ is, of course, not new. The equivalence principle in its general form has, however, not been among the results so obtained.

Our approach is inspired by algebraic local quantum field theory. In addition to the algebra $\mathscr{A}$ of observables we find it necessary to use an algebra $\mathcal{O}$ of observation procedures of which $\mathscr{A}$ is a homomorphic image. The principal difference between these two algebras is the requirement that two local subalgebras $\mathcal{O}\left(R_{1}\right)$ and $\mathcal{O}\left(R_{2}\right)$ associated to nonintersecting space-time regions $R_{1}$ and $R_{2}$ have only a trivial (scalar)

$\star$ Work performed under the auspices of the U.S. Atomic Energy Commission.

$\star \star$ Present address: University of the Negev, Beer-Sheva, Israel.

$\star \star \star$ Present address: C.N.R.S. Marseille, Physique Théorique. 
intersection, while $\mathscr{A}\left(R_{1}\right)$ and $\mathscr{A}\left(R_{2}\right)$ do not, in general, have this relation.

The need for this additional structure arises from the study of external forces and it may be illustrated by classical relativistic mechanics. The usual statement of the principle of special relativity is: The laws of motion are the same in all inertial frames. The mathematical counterpart for particle mechanics [5] is: A solution of the equation of motion is a family of world lines. An element of the Poincaré group has a natural action in transforming this family of world lines. If the set of all solutions is closed with respect to the transformations induced by the Poincare group, then the equations are said to be relativistically covariant.

This definition clearly fails in the presence of external forces because "there is a privileged frame". Yet, the principle of relativity was first applied to particles in external fields. Some results of special relativity, such as the Lorentz contraction of the distance between two particles and the time dilation of decay, remain valid in the presence of external magnetic fields, even when the covariance of the equations of motion fails [2].

Presymmetry $[1,2]$ gives a precise and nontrivial statement of what may be called residual covariance in the presence of external forces. Its conclusions are equally relevant to subsystems of free systems that are under the influence of the forces due to other subsystems. This clarification requires a rather elaborate structure with two algebras instead of the usual theory in terms of differential equations, but for this price one obtains precise statements of such basic principles as local independence $[3,4]$ (or, synonymously, finite signal velocity, or Einstein causality), which are only stated illustratively or allusively in the usual context, and one obtains Newton's second law as a theorem $[2,4]$.

The principles of presymmetry, including the postulate of local acceleration covariance [4], will be used to derive the equivalence principle as a theorem.

The present paper is substantially selfcontained and starts by formulating assumptions on nonmathematical objects and procedures. The mathematical structures are then introduced as fully interpreted images of the nonmathematical objects. Postulates, obtained by idealization or extrapolation of experience, are formulated in Sections $2-5$. The equivalence principle is stated in an operationally verifiable (or at least refutable) form in Section 6. The postulates are realized in Sections 8 and 9 by concrete mathematical objects. Section 10 gives a summary of assumptions. In the remaining sections, the equivalence principle is derived and discussed.

Our results are in substantial agreement with those of Einstein's theory, and the relationship is established in Sections 12-15. 
The abstract algebras $\mathcal{O}$ of observation procedures and $\mathscr{A}$ of observables have representations as algebras of functionals on carrier spaces $\Omega$ of functions $\psi$ on $M$. In the absence of gravitational forces the natural choice for $\mathscr{A}$ is a space of functions $\psi$ on Minkowski space, but gravitational forces make an alternative choice more natural and convenient: a carrier space of functions on a pseudo-Riemannian manifold. In this representation, the identity of our result with the usual curved-space theory (with asymptotic flatness) becomes evident. This result is a more precise version of the conventionalist concept of the relationship between geometry and physics.

\section{The Collections of Observation - and of State-Preparing Procedures}

We consider experiments in laboratories that are not necessarily isolated or shielded from outside influences. It is assumed that the experimenter can control external influences so that they are reproducible for sequences of experiments. The test for a successful control is the satisfaction of certain causality requirements to be given later.

An experiment consists of two procedures: the preparation of a state and the performance of an act of observation that results in a dial reading or printout of a real number. By a slight abuse of language we identify the physical acts of constructing instruments and using them under certain conditions with instructions for performing these acts. It is almost indispensable to assume for the construction of instruments in accordance with blueprints $b$ that distances and time differences be understood in a naive manner, i.e. that Euclidean geometry and constant light velocity be assumed. These conditions may be realized, in the absence of gravitational fields, by suitably shielding the workshop, but for the general case it will be assumed that there exists an asymptotic region where these naive assumptions are justified, and that the instructions call for construction of instruments in this asymptotic region.

A complete procedure is specified by blueprints $b$ and a set $\left\{P_{n}\right\}$ of space-time points at which marks on instruments are to be positioned for the performance, buttons to be pressed or levers to be thrown.

Since the instruments will be used in gravitational fields, we cannot assume that these points $P_{n}$ can be located by the naive use of light rays or meter sticks. We rather assume that there exists a procedure that allows to correct for the bending of light rays and to determine uniquely the positions of points in Minkowski space. The consistency of the theory requires that such a possibility be proved from the assumptions, and this will be done in Section 15.

In a single experiment a state-preparing procedure is followed by an act of observation with a resulting real number $s$. An infinite repetition 
of the experiment produces a sequence $\left\{s_{n}\right\}(n=1,2, \ldots)$ which we assume to be random. An equivalence class $\tilde{s}$ of state-preparing procedures $\left(b,\left\{P_{n}\right\}\right)$ consists of those procedures that have the same mean value $\left\{S_{n}\right\}$ for each observation procedure. An equivalence class $\tilde{\alpha}$ of observation procedures consists of those procedures that create the same expectation value $\left\{\overline{\left.s_{n}\right\}}\right.$ for each state-preparing procedure. We have thus two nonmathematical collections: the collection $\tilde{\mathscr{S}}$ of (equivalence classes of state-preparing procedures, with elements $\tilde{s}$ and the collection $\tilde{\mathcal{O}}$ of (equivalence classes of) observation procedures $\tilde{\alpha}$. The mean values for a given reproducible external field will be designated by $\tilde{E}(\tilde{s}, \tilde{\alpha})$ such that

$$
\tilde{\mathscr{E}}: \tilde{\mathscr{S}} \times \tilde{\mathcal{O}} \rightarrow R
$$

is a map on the cartesian product of the two collections into the reals.

Given a procedure which can be identified with a pair $\left(b,\left\{P_{n}\right\}\right)$ consisting of blueprints $b$ and a set of points $\left\{P_{n}\right\}$ in Minkowski space, an altered instruction $\left(b,\left\{g P_{n}\right\}\right)$ can be obtained by changing all points $P_{n}$ in accordance with a transformation

$$
g: M \rightarrow M \quad\left[P_{n} \mapsto g P_{n}\right]
$$

of Minkowski space $M$. In general, such an altered instruction will not be implementable and will therefore not correspond to an observation procedure. For some transformations $g$, however, the altered instruction corresponds to a feasible procedure and the transformation preserves all equivalence classes. It induces a permutation $\tilde{V}_{g}$ of $\tilde{\mathcal{O}}$.

In particular, a time transformation

$$
P \equiv(\boldsymbol{x}, t) \mapsto(\boldsymbol{x}, t+\tau)
$$

induces a change of each procedure through $\left(b,\left\{P_{n}\right\}\right) \mapsto\left(b,\left\{g P_{n}\right\}\right)$, such that a) the altered instruction is again an observation procedure and b) that each equivalence class of procedures remains an equivalence class under this change. The existence of such transformations is the basic fact that makes physics possible. Indeed, physics compares the results of observations at different instants by the same instrument, and the test for the "sameness" is the agreement between different instruments of the same equivalence class. Experimenters spend most of their time in "checking their instruments", which is a shorthand expression for verifying the preservation of equivalence classes. Note that this property is far more general than time-translation invariance of equations of motion. It is the prime example of presymmetry. 
It will be assumed that all transformations $g$ of the Poincare group $P$ have the same property. This assumption is just a more precise version of the observation that measurement instruments can be translated, rotated and set in uniform motion without being ruined. The test for their continued proper functioning after the motion is accomplished, is the continued agreement of different instruments of the same equivalence class.

The same assumptions for state-preparing procedures are justified by elementary experience. The elements $g$ of $P$ induce permutations $\tilde{W}_{g}$ of $\tilde{\mathscr{S}}$.

We assume that some observation procedures are affected only by events in a region $R$ of Minkowski space. It is not necessary that the instruments be located in $R$, but only that their result be unaffected by changes outside $R$. In addition, we assume that this region $R$ may be a single point $x$ in $M$. (This assumption is natural in the context of classical, but not quantum mechanics.)

A collection $\tilde{\mathcal{O}}_{x}$ of (equivalence classes of point observation procedures is stable under that permutation of $\tilde{\mathcal{O}}$ which is induced by a Lorentz transformation that leaves the point $x$ fixed.

In addition to the usual transformations, we consider a family of groups $\mathscr{M}_{x}$ of acceleration transformations $g$, that leave the points $x$ fixed, i.e. $g \in \mathscr{M}_{x}$ implies $g x=x$. This group has been introduced in Reference 4. It is assumed that each equivalence class of procedures in $\tilde{\mathcal{O}}_{x}$ is preserved under all acceleration transformations induced by accelerations $g \in \mathscr{M}_{x}$. Hence, each acceleration group $\mathscr{M}_{x}$ induces permutations of the collection $\tilde{\mathscr{O}}_{x}$.

Again, this assumption is just a more precise version of the observation that "very small" accelerated laboratories with properly functioning instruments exist - the test for proper functioning being the agreement of printouts of different accelerated instruments belonging to the same equivalence class.

\section{The Algebra $\mathcal{O}$ of Observation Procedures and the Convex Linear Set $\mathscr{S}$ of State-Preparing Procedures}

Classical physics assumes that, in the usual language, two observations can be "performed simultaneously" without disturbing each other. More precisely, a single sample (the product of a single act of state preparation) can be subjected to two observation procedures without interference. The operational meaning of this expression is the equality of mean values of observation results (i) in a sequence of experiments where only one procedure is used on one sample and (ii) in a sequence 
with two observation acts used on a single sample, the state-preparing procedure and the external field being the same in the two sequences. The most frequently used example refers to two simultaneous observation acts such as position- and momentum measurements, but an equally good example is that of two successive position measurements on the same sample.

Of course, this non-interfering nature of two observations is asserted only for some (very gentle) observation procedures, such as position measurements with very soft $X$ rays, but the assumption alleges that such gentle procedures exist in each equivalence class of observation procedures. One can then consider the mean value of the sums of individual outcomes

$$
\lim _{N \rightarrow \infty} \frac{1}{N} \sum_{1}^{N}\left[s_{n}^{\prime}(\tilde{\alpha})+s_{n}^{\prime}(\tilde{\beta})\right]
$$

obtained in such experiments involving two measurements on each sample and assert that it is the sum of the two mean values

$$
\lim _{N \rightarrow \infty} \frac{1}{N} \sum_{1}^{N} s_{n}(\tilde{\alpha})+\lim _{N \rightarrow \infty} \frac{1}{N} \Sigma s_{n}(\tilde{\beta})
$$

obtained in the two sequences involving only one observation $(\tilde{\alpha}$ or $\tilde{\beta})$ on each sample.

This addition of individual outcomes defines operationally a procedure of the equivalence class $(\tilde{\alpha}, \tilde{\beta})_{+}$such that

$$
\tilde{\mathscr{E}}(\tilde{s}, \tilde{\alpha})+\tilde{\mathscr{E}}(\tilde{s}, \tilde{\beta})=\tilde{\mathscr{E}}\left[\tilde{s},(\tilde{\alpha}, \tilde{\beta})_{+}\right] .
$$

This property of the (not ordered) pair $(\tilde{\alpha}, \tilde{\beta})_{+}$suggests that addition be defined. Since it is awkward to add galvanometers and spectrographs, we define a one-one map $\tilde{\Phi}: \tilde{\mathcal{O}} \rightarrow \mathcal{O}$ of non-mathematical procedures $\tilde{\alpha} \in \tilde{\mathcal{O}}$ to their mathematical images $\alpha \in \mathcal{O}$ and similarly a map $\tilde{\Psi}$ of non-mathematical state-preparing procedures $\tilde{S} \in \tilde{\mathscr{S}}$ to their mathematical images $s \in \mathscr{S}$ such that

where

$$
\tilde{\mathscr{E}}(\tilde{S}, \tilde{\alpha})=\mathscr{E}(s, \alpha)
$$

$$
\mathscr{E}: \tilde{\Psi} \tilde{\mathscr{S}} \times \tilde{\Phi} \tilde{\mathcal{O}} \rightarrow R
$$

is a map on the cartesian product $\mathscr{S} \times \mathcal{O}$ into the reals. By assumption, the mathematical image of the pair $(\tilde{\alpha}, \tilde{\beta})_{+}$is the element $\alpha+\beta$ of a linear set $\mathcal{O}$. By assumption, $\mathscr{E}$ is linear in its second argument, so that Eq. (3.3) now reads

$$
\mathscr{E}(s, \alpha)+\mathscr{E}(s, \beta)=\mathscr{E}[s,(\alpha+\beta)]
$$


The same considerations apply to the multiplication of the terms of two sequences of outcomes $s_{n}(\tilde{\alpha})$ and $s_{n}(\tilde{\beta})$ of observation procedures $\tilde{\alpha}$ and $\tilde{\beta}$, if observations are made on the same sample. The products $s_{n}(\tilde{\alpha}) \cdot s_{n}(\tilde{\beta})$ are defined to be outcomes of a new procedure designated by the (not ordered) pair $(\tilde{\alpha}, \tilde{\beta})_{\Lambda}$. The mathematical counterpart of this procedure is the commutative product

$$
\alpha \beta=\beta \alpha=\tilde{\Phi}(\tilde{\alpha}, \tilde{\beta})_{\Lambda} .
$$

Thus, the set $\mathcal{O}$ has the structure of a real Abelian algebra, and each algebraic operation has a well-defined operational counterpart in $\tilde{\mathcal{O}}$.

State-preparing procedures can be composed in a well-known manner [6]. Thereby, the mathematical image $\mathscr{S}$ acquires the structure of a convex linear set.

Composing two instructions in the way discussed above results in a new instruction that contains the sets $\left\{P_{n}\right\}$ of both original instructions. Motion consists in replacing each point $P_{n}$ by $g P_{n}$. Hence, motions impressed before or after composition have the same effect. For those motions that preserve equivalence classes, it follows that a motion $g$ in the non-mathematical collection $\tilde{\mathcal{O}}$ induces a transformation $V_{g} \mathcal{O}$ of the algebra $\mathcal{O}$ that preserves linearity and multiplication. Hence, these motions $g$ create automorphisms of $\mathcal{O}$. Similarly, the motions $g$ of the acceleration group $\mathscr{M}_{x}$ that preserve equivalence classes in $\tilde{\mathcal{O}}_{x}$ induce automorphisms of the subalgebras $\mathcal{O}_{x} \subset \mathcal{O}$.

Similarly, an element $g$ of the Poincaré group induces an automorphism $W_{g}$ of the convex linear set $\mathscr{S}$.

\section{Fields}

In the instruction $\left(b,\left\{P_{n}\right\}\right)$, the ordered set $\left\{P_{n}\right\}$ of points $P_{n}$ of Minkowski space, (where $n$ is in an index set $I$ ), prescribes portions of the world lines of marks on instruments and also specifies space-directions, e.g., by orienting arrows marked on surfaces. The point $x$ and various portions of curves in the neighborhood of $x$ must be included among the instructions for performance of point observation procedures $\tilde{\alpha} \in \tilde{\mathcal{O}}_{x}$. A field at the point $x$ belongs to a particular class of point procedures that specify only families of curves with the same direction at the point $x$, so that the set $\left\{P_{n}\right\}$ can be replaced by $\left\{x, t_{1}, \ldots, t_{m}\right\}$ where $t_{1}, \ldots, t_{m}$ are tangent vectors of families of curves at $x$. The effect of a differentiable transformation $g: M \rightarrow M$ of the form

$$
(g x)_{\mu}=g_{\mu}\left(x_{1}, \ldots, x_{4}\right)
$$


on a tangent vector $t$ at $x$ is

$$
[g t(x)]_{\mu}=\left[\partial(g x)_{\mu} / \partial x_{v}\right] t_{v}(x) .
$$

If $g$ is an element $\Lambda$ of that Lorentz transformation which has $x$ as a fixed point, then the effect of a permutation $\tilde{V}$ on an element $\tilde{\alpha}=\left(b, x, t_{1}, \ldots, t_{m}\right)$ is

$$
\tilde{V}_{\Lambda} \tilde{\alpha}=\left(b, x, \Lambda t_{1}, \ldots, \Lambda t_{m}\right) .
$$

Consider as an example the mass density $\tilde{\varrho}(x)$. In addition to the blueprint $b_{\varrho}$ and the point $x \in M$ the instruction specifies the instantaneous 4-velocity of a mark on the apparatus. If the velocity vector is normalized to $v_{\mu} v^{\mu}=1$, then the space components are velocities $\left(d x_{i} / d t\right)\left(1-v^{2}\right)^{-1 / 2}$, with $i=1-3$. The procedures $\tilde{j}_{i}(x)$ for observation of the current density are specified by a blueprint $b_{j}$ and a space-like vector $a$ that stands for an arrow on the instrument, while the 4-velocity $v$ of a mark on the apparatus is normal to $a$ :

$$
a_{\mu} v^{\mu}=0 .
$$

The electric field intensity $\tilde{E}_{i}(x)$ is measured by a procedure $\left(b_{\tilde{E}}, x, v, t\right)$, where $b_{\tilde{E}}$ is a blueprint, $x$ a point, $v$ a time-like and $t$ a space-like unit vectors, respectively, with obvious operational meaning.

Each permutation $\tilde{V}$ on $\tilde{\mathcal{O}}_{x}$ induces an automorphism $V$ on $\mathcal{O}_{x}$. The simplest automorphism of $\mathcal{O}_{x}$ induced by a Lorentz transformation $\Lambda$ such that $\Lambda x=x$, namely the permutation

$$
(b, x, t) \mapsto \tilde{V}_{\Lambda}(b, x, t)=(b, x, \Lambda t),
$$

is generated by the linear transformation

$$
\tilde{\Phi}(b, x, t) \mapsto V_{\Lambda} \tilde{\Phi}(b, x, t)=\Lambda_{\mathscr{O}} \tilde{\Phi}(b, x, t),
$$

where $\Lambda_{\mathscr{O}}$ is a Lorentz transformation in a 4-dimensional subspace of $\mathcal{O}_{x}$. As a generalization, it is natural to consider the automorphism $V_{A}$ induced by

$$
\left(b, x, t_{1}, \ldots, t_{m}\right) \mapsto \tilde{V}_{\Lambda}\left(b, x, t_{1}, \ldots, t_{m}\right)=\left(b, \Lambda t_{1}, \ldots, \Lambda t_{m}\right)
$$

through

$$
V_{\Lambda} \tilde{\Phi}\left(b, x, t_{1}, \ldots, t_{m}\right)=\Lambda_{\mathscr{O}}^{\otimes m} \tilde{\Phi}\left(b, x, t_{1}, \ldots, t_{m}\right)
$$

where $\Lambda_{\mathscr{O}}^{\otimes m}$ is the linear transformation induced by $\Lambda_{\mathscr{O}}$ on a subspace of a $4^{m}$-dimensional tensor space in $\mathcal{O}_{x}$.

Fields belong to the class of point observation procedures that have these particularly simple transformation properties. In particular, we assume that a specific field $\left\{\tilde{\phi}_{i}\right\}$ is a subcollection of observation 
procedures $\tilde{\phi}_{i}\left(b_{i \lambda}, x, t_{1}, \ldots, t_{m_{i}}\right)$ whose images $\tilde{\Phi} \tilde{\phi}_{i} \in \mathcal{O}_{x}$ span an irreducible representation space of the Lorentz group that is a subspace of the $4^{m}$-dimensional space mentioned before. A number of different blueprints $b_{i \lambda}$ are always associated to a given field $\tilde{\phi}_{i}$. In the simplest case of a 4-dimensional representation, we found that two blueprints are needed, one for the time-like and the other for the space-like vectors in the representation space, so that $\lambda=1,2$. Similarly, the electromagnetic field requires two distinct blueprints $(\lambda=1,2)$ : one for the electric and one for the magnetic field. It is assumed naturally that no two blueprints $b_{i, \lambda}$ and $b_{j, \mu}$ are equal unless $i=j$ and $\mu=\lambda$.

A particular class of observation procedures is called intrinsic or canonical $[1,2]$ because their expectation values are independent of the equations of motion and of any external fields. More precisely, let $x \in h$ be a point in a space-like hyperplane $h \subset M$, and let $\tilde{\mathscr{S}}_{h}$ be that subset of state-preparing procedures which creates states (objects of measurements) at the instant specified by $h$. Then if $\tilde{s} \in \tilde{\mathscr{S}}_{h}$ and $x \in h$, a canonical observation procedure $\tilde{\alpha} \in \tilde{\mathcal{O}}_{x}$ is required to have the same expectation value $\tilde{\mathscr{E}}(\tilde{s}, \tilde{\alpha})$ for all $\tilde{\mathscr{E}}$ in the collection $\{\tilde{\mathscr{E}}\}$ of expectations. Examples of such procedures are current density, charge density, and other fields, but not derivatives of these quantities. In particle physics, position and momentum are canonical, but velocity and acceleration are not. To summarize, a field is a subcollection $\tilde{\phi}_{i}=\left\{\left(b_{i \lambda}, x, t_{1}, \ldots, t_{m}\right)\right\}$ of observation procedures at a fixed point $x$ and for a fixed $i$ but with variable $\lambda$ and $t_{l}$, and it has the following properties.

1. A field component depends on the collection $\left\{P_{n}\right\}$ of events in the instructions only through tangent vectors $t_{1}, \ldots, t_{m_{i}}$ and the point $x$, i.e., $\tilde{\phi}_{i \lambda}=\left\{\left(b_{i \lambda}, x, t_{1}, \ldots, t_{m}\right)\right\}$. The collection is closed with respect to the Lorentz transformations that leave $x$ fixed; i.e.,

$$
\tilde{V}_{\Lambda}\left(b_{i \lambda}, x, t_{1}, \ldots, t_{m}\right)=\left(b_{i \lambda}, x, \Lambda t_{1}, \ldots, t_{m}\right) \in \tilde{\phi}_{i \lambda}
$$

2. The images $\left\{\tilde{\Phi} \tilde{\phi}_{i}\right\}$ in $\mathcal{O}_{x}$ span a subspace of a $4^{m}$-dimensional tensor space in $\mathcal{O}_{x}$. A Lorentz transformation $\Lambda$ induces the linear transformation

$$
\begin{aligned}
\tilde{\Phi}\left(b_{i}, x, t_{1}, \ldots, t_{m_{i}}\right) & \\
& \mapsto \tilde{\Phi} \tilde{V}_{\Lambda}\left(b_{i}, x, t_{1}, \ldots, t_{m_{\imath}}\right)=\Lambda_{\mathscr{O}}^{\otimes m_{\imath}} \tilde{\Phi}\left(b_{i}, x, t_{1}, \ldots, t_{m_{i}}\right) .
\end{aligned}
$$

The image $\tilde{\Phi} \tilde{\phi}_{i}$ of a field spans an irreducible representation space of the Lorentz group in $\mathcal{O}_{x}$.

3. A field is canonical. That is, if $x \in h$ and $\tilde{\mathscr{S}}_{h}$ is the collection of state-preparing procedures $\tilde{s}$ emitting samples at the instant $h$, then the expectation value $\tilde{\mathscr{E}}(\tilde{s}, \tilde{\phi})$ is the same for all expectations $\tilde{\mathscr{E}} \in\{\tilde{\mathscr{E}}\}$ if $\tilde{s} \in \tilde{\mathscr{S}}_{h}$. 


\section{Causality and Local Subalgebras}

Causality requires that the knowledge of all expectation values of observation procedures at an instant $h$ provide full predictive power. For each $\tilde{\beta} \in \tilde{\mathcal{O}}$, there exists a unique procedure $\tilde{\alpha}(\tilde{\mathscr{E}}) \in \tilde{\mathcal{O}}_{h}$ such that

$$
\tilde{\mathscr{E}}(\tilde{s}, \tilde{\alpha})=\tilde{\mathscr{E}}(\tilde{s}, \tilde{\beta}), \tilde{s} \in \tilde{\mathscr{S}}, \tilde{\alpha} \in \tilde{\mathcal{O}}_{h}, \tilde{\beta} \in \tilde{\mathcal{O}} .
$$

This version of causality is only apparently different from the more familiar assertion that the expectation value of any observation procedure can be calculated from those of the instantaneous procedures at an instant $h$. In our definition of procedures, the result of any such calculation is, in turn, the expectation value of an instant procedure.

In Section 4 we considered canonical procedures that are universal in the sense that their expectation values are independent of external forces. The canonical procedures should be sufficient for prediction. Otherwise, how could one compare the time-development of the same initial state with different external forces? Hence, we are led to postulate that causality holds for a restricted set $\tilde{\mathcal{O}}_{h c} \subset \tilde{\mathcal{O}}_{h}$ which is the inverse image $\tilde{\Phi}^{-1} \mathcal{O}_{h c}$ of a canonical algebra $\mathcal{O}_{h c}$. The stronger version of causality [2] claims that for each $\tilde{\beta} \in \tilde{\mathcal{O}}$ and $\tilde{s} \in \tilde{\mathscr{T}}_{h}$, there exists a procedure $\tilde{\alpha}(\tilde{\mathscr{E}}) \in \tilde{\mathcal{O}}_{h c}$ such that

$$
\tilde{\mathscr{E}}(\tilde{s}, \tilde{\alpha})=\tilde{\mathscr{E}}(\tilde{s}, \tilde{\beta}), \tilde{s} \in \tilde{\mathscr{T}}_{h}, \tilde{\alpha} \in \tilde{\mathcal{O}}_{h c}, \tilde{\beta} \in \tilde{\mathcal{O}},
$$

and $\tilde{\alpha}$ is independent of $\tilde{s}$.

In Section 4 the fields $\tilde{\phi}(x)$ were defined as canonical procedures. Since the experience of classical field theories is that the fields have the canonical property of providing full predictive power, we assume that $\mathcal{O}_{h c}$ is the algebra generated (in a sense to be defined presently) by the fields on a space-like hyperplane $h$, i.e.,

$$
\mathcal{O}_{h c}=\overline{\bigcup_{x \in h} \tilde{\Phi} \tilde{\phi}(x)} .
$$

The counterpart of causality in $\mathcal{O}$ is a statement concerning the timeindependence of what may be called a natural law. Suppose that the equivalence (indistinguishability) of two observation procedures $\alpha, \beta \in \mathcal{O}$ has been established for a given $\varepsilon$ by measuring their expectation values $\mathscr{E}(s, \alpha)$ and $\mathscr{E}(s, \beta)$ with respect to all state-preparing procedures at an instant $h$, i.e., $s \in \mathscr{S}_{h}$. We postulate that this equivalence is valid for all states, prepared at any instant. That is, the finding $\mathscr{E}(s, \gamma)=0$ for all $s \in \mathscr{S}_{h}$ with a fixed $h$ implies $\mathscr{E}(r, \gamma)=0$ for $r \in \mathscr{S}$.

The algebra $\mathcal{O}$ of observation procedures has a natural topological structure: Two elements $\alpha$ and $\beta \in \mathcal{O}$ that induce nearby real expectation values $\mathscr{E}(s, \alpha)$ and $\mathscr{E}(s, \beta)$ for all $s \in \mathscr{S}$ must be considered as nearby in the 
topology. This requirement restricts the class of possible topologies of $\mathcal{O}$, but the precise choice is made in Section 10 on grounds of mathematical convenience. Given a subset $\mathscr{L} \subset \mathcal{O}$, the set of all finite polynomials of elements of $\mathscr{L}$, together with the topological limits of sequences in this set, will be called the topological closure $\mathscr{L}$ of the subset $\mathscr{L}$.

From point algebras $\mathcal{O}_{x} \subset \mathcal{O}$, we can define local subalgebras $\mathcal{O}(R)$ as the closure

$$
\mathcal{O}(R)=\overline{\bigcup_{x \in R} \mathcal{O}_{x}}
$$

if $R$ is an open set. If $R$ is not open (e.g., if it is a point $x$ or a hyperplane $h$ ), the local algebra $\mathcal{O}(R)$ is defined as the limit of convergent sequences $\mathcal{O}\left(R_{n}\right), R_{n} \rightarrow R$, if this limit exists. In particular, $\mathcal{O}_{x}$ and $\mathcal{O}_{h c}$ are such limits, but the canonical subalgebras $\mathcal{O}_{h c}$ are not local algebras. The requirements of this collection of local algebras are as follows.

1. The intersection $\mathcal{O}\left(R_{1}\right) \cap \mathcal{O}\left(R_{2}\right)$ of two local algebras associated to nonintersecting regions $R_{1}$ and $R_{2}$ is trivial: it consists only of multiples of the unit $I$. That is,

$$
\mathcal{O}\left(R_{1}\right) \cap \mathcal{O}\left(R_{2}\right)=\{c I\} \text {. }
$$

2. The topological closure $\overline{\mathcal{O}\left(R_{1}\right) \cup \mathcal{O}\left(R_{2}\right)}$ of the union of two local algebras $\mathcal{O}\left(R_{1}\right)$ and $\mathcal{O}\left(R_{2}\right)$ is the local algebra associated to $R_{1} \cup R_{2}$. That is

$$
\mathcal{O}\left(R_{1}\right) \cup \mathcal{O}\left(R_{2}\right)=\mathcal{O}\left(R_{1} \cup R_{2}\right)
$$

3. The topological closure of the union of all local subalgebras is $\mathcal{O}$. That is,

$$
\overline{\bigcup_{R \subset M} \mathcal{O}(R)}=\mathcal{O}
$$

\section{Operational Statement of the Equivalence Principle}

We are now ready to state the first part of the equivalence principle. It states that for every expectation $\tilde{\mathscr{E}} \in\{\tilde{\mathscr{E}}\}$, every point $x$ in Minkowski space, and every element $g$ of the acceleration group $\mathscr{M}_{x}$, there exists another expectation $\tilde{\mathscr{E}}_{g} \in\{\tilde{\mathscr{E}}\}$ such that the effect of acceleration on the collection $\tilde{\mathcal{O}}_{x}$ of point observation procedures is compensated. That is

$$
\exists \tilde{\mathscr{E}_{g}} \mid \tilde{\mathscr{E}} g(\tilde{s}, \tilde{\alpha})=\tilde{\mathscr{E}}\left(\tilde{s}, \tilde{V}_{g} \tilde{\alpha}\right), \tilde{\alpha} \in \tilde{\mathcal{O}}_{x}, \tilde{s} \in \tilde{\mathscr{S}}
$$

The principle asserts the existence of an external force that (on being switched on) creates the same effect for any state as the physical act of accelerating all observation apparatus belonging to $\tilde{\mathcal{O}}_{x}$. 
The equivalence principle is frequently stated as a principle of ignorance: It is impossible to find out by local experiments whether a change from a given situation (expectation) has occured because the external field has been altered or because an acceleration has induced a relabeling (permutation) of the instructions for observations. An explicit form of this principle must refer to all possible observation procedures as does Eq. (6.1) and not only to distance - and time - delay measurements as do the usual formulations.

A frequent question in books on relativity concerns the degree $\partial^{n} / \partial x^{n}$ of the derivative of an observable that is meant to be covered by the equivalence principle - the idea being that the $(n+1)$ th derivative refers to a somewhat larger laboratory than the $n$th derivative does. Such questions are legitimate if the method of measurement uses point measurements at two nearby points in order to determine a derivative. This is not the case for our observation procedures. An accelerometer is an instrument quite different from two velocity meters at nearby points, although a sequence of observations of the latter type will approximate the results of the former. Thus the question of smallness does not have a vexing quality in our scheme. All point procedures are dealt with on the same level.

A loose paraphrase of our statement is that inertial forces are equivalent to suitably chosen gravitational forces, but many authors $[7,8]$ consider this statement to be empirically incorrect. Coriolis forces, the argument runs, are inertial forces but cannot be replaced by gravitational forces; in the Newtonian limit, the latter are velocity-independent, the former are not.

The resolution of the discrepancy lies in the narrowly restricted choice of acceleration transformations provided by our group $\mathscr{M}_{x}$ of meromorphisms. In contrast to the immense group of coordinate transformations used in the usual formulation, our group acts strictly on observation procedures at one point and does not contain the more general space-time transformations that induce Coriolis forces.

\section{Inferences from Assumptions}

Consider the kernel

$$
K(\mathscr{E})=\{\alpha \in \mathcal{O} \mid \mathscr{E}(s, \alpha)=0 \text { for all } s \in \mathscr{S}\} .
$$

It is easily seen that $K(\mathscr{E})$ is an ideal.

We now discuss the quotient algebra $\mathcal{O} / K(\mathscr{E})$ and show that it is isomorphic to a subalgebra $\mathcal{O}_{s} \subset \mathcal{O}_{h} \subset \mathcal{O}$, where $\mathcal{O}_{h}$ is the algebra associated to a space-like hyperplane $h$. It follows from the assumption of weak causality (5.1) that for every $\beta \in \mathcal{O}$ there is an $\alpha \in \mathcal{O}_{h}$ such that $\beta-\alpha \in K(\mathscr{E})$. 
Thus, every element $\beta \in \mathcal{O}$ can be written in the form

$$
\beta=\alpha+k, \quad \alpha \in \mathcal{O}_{h}, \quad k \in K(\mathscr{E}) .
$$

By definition, an element $\gamma \in \mathcal{O} / K(\mathscr{E})$ has the form

$$
\gamma=\beta+K(\mathscr{E}), \quad \beta \in \mathcal{O},
$$

and by Eq. (7.1), this gives

$$
\gamma=\alpha+K(\mathscr{E}), \quad \alpha \in \mathcal{O}_{h} .
$$

We now define a homomorphism on $\mathcal{O} / K(\mathscr{E})$ into $\mathcal{O}_{h}$, simply by

$$
\mathcal{O} / K(\mathscr{E}) \ni \gamma=\alpha+K(\mathscr{E}) \mapsto \alpha \in \mathcal{O}_{h} \text {. }
$$

It is easily seen that this map preserves the algebraic structure. On the other hand, the range of this map is not necessarily the entire algebra $\mathcal{O}_{h}$, since weak causality does not imply that we must exhaust all of $\mathcal{O}_{h}$ in order to obtain the ideal $K(\mathscr{E})$. Clearly, $\mathcal{O} / K(\mathscr{E})$ is isomorphic to a subalgebra $\mathcal{O}_{s} \subset \mathcal{O}_{h}$, defined simply as the range of the map. It will be shown now that $\mathcal{O}_{s}=\mathcal{O}_{h c}$, where $\mathcal{O}_{h c}$ is the canonical subalgebra of $\mathcal{O}_{h}$.

By definition $\mathcal{O}_{h c} \subset \mathcal{O}_{h}$ is canonical, if for all pairs $\mathscr{E}_{1}, \mathscr{E}_{2} \in\{\mathscr{E}\}$ and $\alpha \in \mathcal{O}_{h c}$,

$$
\mathscr{E}_{1}(s, \alpha)=\mathscr{E}_{2}(s, \alpha), \quad s \in \mathscr{S}_{h}
$$

It has been assumed in Section 5 that

$$
\mathscr{E}(s, \alpha)=0 \text { for all } s \in \mathscr{S}_{h}
$$

implies that

$$
\mathscr{E}(r, \alpha)=0 \text { for all } r \in \mathscr{S} .
$$

Lemma 1. The only element of $\mathcal{O}_{\text {hc }}$ that belongs to $K(\mathscr{E})$ is 0 .

Proof. $\alpha \in K(\mathscr{E})$, and therefore

$$
\mathscr{E}(s, \alpha)=0 \text { for all } s \in \mathscr{S} \supset \mathscr{S}_{h} .
$$

But because of the canonicity,

$$
\mathscr{E}(s, \alpha)=0 \quad \text { for all } \quad s \in \mathscr{S}_{h} \quad \text { and all } \mathscr{E} \in\{\mathscr{E}\}
$$

and by Eq. (7.6) this holds for all $s \in \mathscr{S}$.

Then, by mutual separation, $\alpha=0$.

Lemma 2. $\mathcal{O}_{h c}$ is the minimal subalgebra of $\mathcal{O}_{h}$ for which strong causality is valid. In other words, there is no proper subalgebra $\mathcal{O}_{s} \subset \mathcal{O}_{h c}$ satisfying the axioms of strong causality. 
Proof. Assume that the converse is true, and let $\beta \in \mathcal{O}_{h c}$ but $\beta \notin \mathcal{O}_{s}$. Then there exists an element $\alpha \in \mathcal{O}_{s}$ such that $\beta-\alpha \in K(\mathscr{E})$. But $\beta-\alpha \in \mathcal{O}_{h c}$ and by Lemma $1, \beta-\alpha=0$. Therefore $\beta=\alpha \in \mathcal{O}_{\mathrm{s}}$, contrary to the assumption. Now we can prove

Theorem 1. The quotient $\mathcal{O} / K(\mathscr{E})$ is isomorphic to $\mathcal{O}_{\text {hc }}$. That is,

$$
\mathcal{O} / K(\mathscr{E}) \cong \mathcal{O}_{h c}
$$

Proof. By the definition of the quotient algebra, and by strong causality, every element $\gamma$ of $\mathcal{O} / K(\mathscr{E})$ is expressed as

$$
\gamma=\alpha+K(\mathscr{E}), \quad \alpha \in \mathcal{O}_{h c} .
$$

From Lemma 2, we have

$$
\mathcal{O} / K(\mathscr{E})=\left\{\alpha+K(\mathscr{E}), \alpha \in \mathcal{O}_{h c}\right\}
$$

and hence the map $\mathcal{O} / K(\mathscr{E}) \rightarrow \mathcal{O}_{h c}$ defined by $\alpha+K(\mathscr{E}) \mapsto \alpha$ is bijective and preserves the algebraic structure, i.e., it is an isomorphism.

\section{Realization of the Algebra $\mathcal{O}$ of Observation Procedures and of the Algebra $\mathscr{A}$ of Observables}

In classical mechanics, the algebra of observables is an Abelian algebra of real-valued functions. By analogy, the algebras $\mathcal{O}$ and $\mathscr{A}$ for classical fields are naturally realized as algebras of real functionals on function spaces (carrier spaces) $\Omega$ and $\omega$, respectively. If fields $\tilde{\phi}_{i, x}$ at a point are to have images in $\mathcal{O}$, these must be unbounded elements, because the physical meaning of a field at a point is incompatible with a bound. Hence, the algebra $\mathcal{O}$ must be a topological algebra but not a normed one.

While the natural representation of normed Abelian algebras is obtained by Gelfand's representation theorem by functions on a nonlinear carrier space [9], the natural space of functions is linear, as is the phase space of classical mechanics. In classical mechanics, the canonical observables $q_{i}$ and $p_{i}$ are linear functions on phase space. By analogy, the canonical functionals $\tilde{\Phi} \tilde{\phi}_{i, x, t_{1} \ldots t_{m_{i}}}=F_{i, x, t_{1} \ldots t_{m_{2}}}$ which, by a slight abuse of language, we also call fields, are assumed to be linear functions on $\Omega$. We assume that $\Omega$ is the linear space spanned by $N$ subspaces of irreducible covariant-tensor-valued functions $\phi_{i}(i=1$ to $N)$ on Minkowski space. More explicitly, let

$$
\begin{gathered}
\phi_{i}: M \rightarrow T_{i} ;\left[M \ni x \mapsto v_{i}(x)\right] \\
v_{i}(x): V^{\otimes m_{i}} \rightarrow R ;\left[V^{\otimes m_{\imath}} \ni\left(t_{1}, t_{2} \ldots t_{m_{\imath}}\right) \mapsto \phi_{i}\left(t_{1}, t_{2} \ldots t_{m_{i}}, x\right)\right] .
\end{gathered}
$$


That is, $\phi_{i}$ is a map on Minkowski space into the $m_{i}$-dimensional real linear vector space $T_{i}$ of covariant tensors $v_{i}$ of rank $m_{i}$. These are defined [10] as multilinear forms on the $m_{i}$-fold Cartesian product $V^{\otimes m_{i}}$ of 4-dimensional real vector spaces $V$. The range of $\phi_{i}$ is a subspace of $T_{i}$, namely a space irreducible with respect to the representation of the Lorentz group induced by the Lorentz transformation

$$
L: V \rightarrow V
$$

of the underlying 4-dimensional vector space $V$. Alternatively, one can consider some elements of $\Omega$ as functions on the Cartesian product of $M$ and the vector space $V^{\otimes m_{i}}$ into the reals, i.e.,

$$
\begin{gathered}
M \times V^{\otimes m_{i}} \rightarrow R ; \\
\left(x, t_{1}, t_{2} \ldots t_{m_{i}}\right) \mapsto \phi_{i}\left(t_{1}, t_{2} \ldots t_{m_{i}}, x\right),
\end{gathered}
$$

and the general element of $\Omega$ as a real linear combination of these functions, with $i=1, \ldots, N$. The fields are defined by

$$
F_{i, x, t_{1}, t_{2} \ldots t_{m_{i}}}\left(\phi_{j}\right)=\delta_{i j} \phi_{j}\left(t_{1}, t_{2} \ldots t_{m_{j}}, x\right) \text {. }
$$

If one introduces an orthonormal basis $\left\{l^{\mu}\right\}(\mu=1, \ldots 4)$ in $V$, so that

then

$$
t_{i}=t_{i \mu} l^{\mu},
$$

$$
F_{i, x, t_{1}, t_{2} \ldots t_{m_{i}}}\left(\phi_{i}\right)=\phi_{\mu_{1}, \mu_{2}, \ldots \mu_{m_{i}}}(x) t_{\mu_{1}} t_{\mu_{2}} \ldots t_{\mu_{m_{i}}} \text {, }
$$

where $\phi_{\mu_{1}, \mu_{2} \ldots \mu_{m_{i}}}$ are the usual covariant tensor components

$$
\phi_{\mu_{1}, \mu_{2} \ldots \mu_{m_{i}}}(x)=\phi\left(l^{\mu_{1}}, l^{\mu_{2}}, \ldots, l^{\mu_{m_{i}}}, x\right) .
$$

For convenience rather than for compelling physical reasons, we assume the elements $\phi_{i}\left(t_{1}, t_{2}, \ldots, t_{m_{i}}\right)$, of $\Omega$ to be once differentiable $\left(C^{1}\right)$ and bounded. With an orthonormal basis, and components $\phi_{\mu_{1} \ldots \mu_{m_{i}}}(x)$, the tensor fields $\phi_{i}$ have the usual aspect of classical fields.

For convenience, the topology of $\Omega$ is assumed to be given by the norm

$$
\left\|\phi_{i}\right\|=\operatorname{Sup}_{\substack{x \in M \\ l^{\mu} \in V}}\left(\left|\phi_{i, \mu_{1}, \ldots, \mu_{m_{i}}}(x)\right|+\sum_{j=1}^{4}\left|\partial \phi_{i, \mu_{1}, \ldots, \mu_{m_{i}}}(x) / \partial x_{j}\right|\right)
$$

where $\left|\mu^{\mu} l_{\mu}\right|=1$ and

$$
\left\|\Sigma \lambda_{i} \phi_{i}\right\|=\left(\Sigma \lambda_{i}^{2}\left\|\phi_{i}\right\|^{2}\right)^{1 / 2}
$$

under which $\Omega$ is a Banach space.

The functionals $F \in \mathcal{O}$ are assumed to be real and continuous, equipped with the $c$-topology which has been used and discussed in 
Ref. [3] and [4]. This topology does not exclude unbounded elements. The canonical subalgebra $\mathcal{O}_{0 c}$ is generated by the representatives $F_{i, x}$ of the fields $\tilde{\phi}_{i, x}$ for the points $x:(0, x)$. Since the algebra $\mathscr{A}$ of observables is isomorphic to $\mathcal{O}_{0 c}$, it can be generally represented as the algebra of real, continuous functionals on the linear space $\omega$ of tensor-valued functions $\psi^{i}$ on $R^{3}$. The topologies of $\omega$ and $\mathscr{A}$ are those inherited from $\Omega$ and $\mathcal{O}$, respectively.

The local algebras $\mathcal{O}(R) \subset \mathcal{O}$ and $\mathscr{A}(R)$ in $\mathscr{A}$ can be shown to have the properties postulated in Sections $2-6$. The relevant theorems are found in Ref. [3] and [4].

\section{Realization of Motion-Induced Automorphisms}

The algebras $\mathcal{O}$ and $\mathscr{A}$ have large groups of automorphisms, but our interest is restricted to the motion-induced subgroup, i.e., those induced by the permutations

$$
\left(b,\left\{P_{n}\right\}\right) \mapsto\left(b,\left\{g P_{n}\right\}\right)
$$

in the hardware collection $\tilde{\mathcal{O}}$, introduced in Section 2.

By analogy with the change of operating instructions described in Eqs. (4.1) and (4.2), a differentiable transformation $g$ of Minkowski space induces a homeomorphism $h: \Omega \rightarrow \Omega$ of the carrier space through

$$
h_{g}: \phi_{i}\left(t_{1}, \ldots t_{m_{i}}, x\right) \mapsto \phi_{i}\left(g t_{1}, \ldots g t_{m_{i}}, g x\right),
$$

where the orthonormal components $t^{\mu}$ of the vectors $t_{l}$ transform through

$$
(g t)^{\mu}=\frac{\partial(g x)^{\mu}}{\partial x^{v}} t^{v} \quad(\mu, v=1, \ldots, 4) .
$$

This homeomorphism induces an automorphism $V_{g}: \mathcal{O} \rightarrow \mathcal{O}$ through

$$
F\left(\phi_{i}\right) \mapsto F\left(h_{g} \phi_{i}\right) \equiv\left(V_{g} F\right)\left(\phi_{i}\right) .
$$

The motion-induced automorphisms of $\mathcal{O}$ and of $\mathcal{O}_{x}$ will now be induced through homeomorphisms of $\Omega$. The Poincaré group $\mathscr{P}$ of transformations of $M$ is mapped into the group Perm $(\mathcal{O})$ of motion-induced permutations of $\tilde{\mathcal{O}}$ through Eq. (9.1), and this group is mapped into the group $\operatorname{Aut}(\mathcal{O})$ of the automorphisms of $\mathcal{O}$. The same result is obtained by first mapping $\mathscr{P} \cong \operatorname{Aut}(M)$ into the group $\operatorname{Hom}(\Omega)$ of the homeomorphisms of $\Omega$ through Eq. (9.2), and following it by a map of these homeomorphisms into $\operatorname{Aut}(\mathcal{O})$ through Eq. (9.4). This is illustrated by the commutative diagram of Fig. 1. For an element $(\Lambda, a)$ of the Poincaré group, Eq. (9.2) reads

$$
\phi_{i}\left(t_{1}, \ldots, t_{m_{i}}, x\right) \mapsto \phi_{i}\left(\Lambda t_{1}, \ldots, \Lambda t_{m_{i}}, \Lambda x+a\right) .
$$




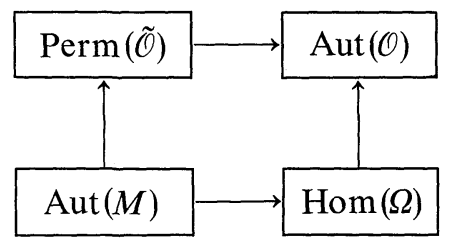

Fig. 1

Since $\phi_{i}$ is a multilinear function of the $m_{i}$ vectors $t_{l}$, and since it is an element of an irreducible representation of the Lorentz group, the righthand side of Eq. (9.5) has the form

$$
S_{\mu_{1}, \mu_{2}, \ldots, \mu_{m_{i}}}(\Lambda) \phi_{i}\left(l^{\mu_{1}}, \ldots, l^{\mu_{m_{i}}}, \Lambda x+a\right),
$$

where $S_{\mu_{1} \ldots \mu_{m_{i}}}$ is an element of an irreducible representation of the Lorentz group. This is the usual transformation law of relativistic fields. Thus, the tensor fields of the conventional field theory occur in our scheme as elements of a carrier space $\Omega$. While this is a natural choice, it is by no means unique, since a given algebra $\mathcal{O}$ has infinitely many isomorphic representations by functionals on carrier spaces. Questions concerning the smoothness or multiplicity of these fields have in the present scheme no intrinsic standing. The choice is one of mathematical convenience.

We now turn to the realization of automorphisms of the point algebra $\mathcal{O}_{x}$ associated to the point $x$, induced by the group $\mathscr{M}_{x}$ of meromorphisms of $M$. To apply our construction to the acceleration group $\mathscr{M}_{x}$, a convention is necessary. Since the acceleration transformations $g$ are in general not differentiable on the light cone, and leave the outside of the light cone fixed, it is natural to require that the derivatives $\partial g / \partial x_{\mu}$ be limits of derivatives taken inside the light cone. Since these motions in $\mathscr{M}_{x}$ induce only permutations of $\tilde{\mathcal{O}}_{x}$, the automorphisms induced are those of $\mathcal{O}_{x}$ and not of $\mathcal{O}$. In other words, while any transformation $g$ induces an automorphism of $\mathcal{O}$ via $\operatorname{Hom}(\Omega)$, it is only for its restriction to $\mathcal{O}_{x}$ that the diagram of Fig. 2 is commutative.

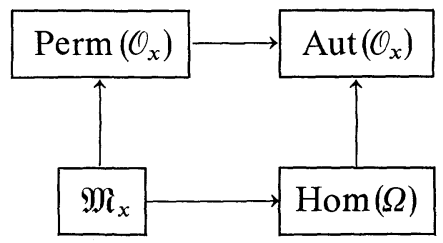

Fig. 2 


\section{Summary of Assumptions}

1. A procedure (or, with some abuse of language, an instruction for its performance) is a pair $\left(b,\left\{P_{n}\right\}\right)$ consisting of a blueprint $b$ and an ordered set of points $P_{n}$ in Minkowski space $M$. Two nonmathematical collections, $\tilde{\mathscr{S}}$ and $\tilde{\mathcal{O}}$ of equivalence classes of state-preparing and of observations procedures, respectively, are considered.

2. The results $s_{n}(\tilde{\alpha})$ of letting observation acts $\tilde{\alpha}$ follow state-preparing acts $\tilde{s}$ on a given physical system with a given external force, are real numbers. The set of mean values associated to a given physical system and external force

$$
\tilde{\mathscr{E}}(\tilde{s}, \tilde{\alpha})=\lim _{N \rightarrow \infty} \frac{1}{N} \sum_{1}^{N} s_{n}(\tilde{\alpha}), \quad(\tilde{s} \in \tilde{\mathscr{S}}, \tilde{\alpha} \in \tilde{\mathcal{O}})
$$

is a member of the collection $\{\tilde{\mathscr{E}}\}$ of expectations.

3. There are two bijections

$$
\tilde{\Phi}: \tilde{\mathcal{O}} \rightarrow \mathcal{O} \quad \text { and } \quad \tilde{\Psi}: \tilde{\mathscr{S}} \rightarrow \mathscr{S}
$$

on the nonmathematical collections of observation and of statepreparing procedures onto the Abelian topological algebra $\mathcal{O}$ and onto the convex linear set $\mathscr{S}$, respectively, and a collection $\{\mathscr{E}\}$ of maps $\mathscr{E}: \mathscr{S} \times \mathcal{O} \rightarrow R$ on the Cartesian product $\mathscr{S} \times \mathcal{O}$ into the reals, such that $\tilde{\mathscr{E}}(\tilde{s}, \tilde{\alpha})=\mathscr{E}(\tilde{\Psi} \tilde{s}, \tilde{\Phi} \tilde{\alpha})$. The map $\mathscr{E}$ is linear on $\mathscr{S}$ and on $\mathcal{O}$.

4. The elements $g$ of the Poincare group, acting on points $P$ of $M$ by $P \mapsto g P$, induce permutations

and

$$
\tilde{V}_{g}: \tilde{\mathcal{O}} \rightarrow \tilde{\mathcal{O}} \quad\left[\left(b,\left\{P_{n}\right\}\right) \mapsto\left(b,\left\{g P_{n}\right\}\right)\right]
$$

$$
\tilde{W}_{g}: \tilde{\mathscr{S}} \rightarrow \tilde{\mathscr{S}}\left[\left(b,\left\{P_{n}\right\}\right) \mapsto\left(b,\left\{g P_{n}\right\}\right]\right.
$$

of $\tilde{\mathcal{O}}$ and $\tilde{\mathscr{S}}$, respectively, and these induce automorphisms

$$
V_{g}: \mathcal{O} \rightarrow \mathcal{O} \text { and } W_{g}: \mathscr{S} \rightarrow \mathscr{S}
$$

of $\mathcal{O}$ and $\mathscr{S}$, respectively, through the natural maps

and

$$
\tilde{\Phi} \tilde{V}_{g} \tilde{\alpha}=V_{g} \tilde{\Phi} \tilde{\alpha} \quad(\alpha \in \tilde{\mathcal{O}})
$$

$$
\tilde{\Psi} \tilde{W}_{g} \tilde{s}=W_{g} \tilde{\Psi} \tilde{s} \quad(\tilde{s} \in \tilde{\mathscr{S}}) \text {. }
$$

5. For each subcollection $\tilde{\mathcal{O}}_{x}$ of point observation procedures associated to $x \in M$, there is an acceleration group $\mathscr{M}_{x}$ of transformations (meromorphisms) of Minkowski space that has $x$ as a fixed point, and its elements induce permutations $\tilde{V}_{g}$ of $\tilde{\mathcal{O}}_{x}$ through

$$
\tilde{\alpha}=\left(b,\left\{P_{n}\right\}\right) \mapsto \tilde{V}_{g} \tilde{\alpha}=\left(b,\left\{g P_{n}\right\}\right)\left(\tilde{\alpha} \in \tilde{\mathcal{O}}_{x}\right) .
$$


These induce, in turn, automorphisms $V_{g}$ of the point subalgebra $\tilde{\Phi} \tilde{\mathcal{O}}_{x}=\mathcal{O}_{x} \subset \mathcal{O}$. The acceleration group $\mathscr{M}_{x}$ is defined in reference 4.

6. A field $\tilde{\phi}_{i, x}$ at a point $x$ is a subcollection of $\tilde{\mathcal{O}}_{x}$ with the following properties.

a) The set $\left\{P_{n}\right\}$ of events in the instruction specifies only equivalence sets of curves with the same tangent vector, so that $\left\{P_{n}\right\}$ can be replaced by a finite set $t_{1}, \ldots, t_{m}$ of vectors in a 4-dimensional real vector space $V$, and the point $x$. The vectors $t_{l}$ are assumed normalized to $|t|=1$ in the Minkowski pseudometric. The field $\tilde{\phi}_{i, x}$ is an equivalence class of instructions of the form

$$
\begin{aligned}
\tilde{\mathcal{O}}_{x} \supset \tilde{\phi}_{i, x} & =\left\{\left(b_{i \lambda}, x, t_{1} \ldots t_{m_{l}}\right)\right\} \\
\lambda & =1, \ldots, k_{i}, \\
t_{1}, \ldots t_{m_{l}} & \in V,\left|t_{l}\right|=1 .
\end{aligned}
$$

The index $\lambda$ that, along with the field label, labels the blueprint, depends only on the time- or space-like nature of the tangent vectors $t_{l}$, and it is therefore fixed under Lorentz transformations.

b) The collection $\tilde{\phi}_{i, x}$ is closed with respect to those Lorentz transformations $\Lambda$ that have $x$ as a fixed point. That is,

$$
\tilde{V}_{\Lambda}\left(b_{i \lambda}, x, t_{1}, \ldots, t_{m_{i}}\right)=\left(b_{i \lambda}, x, \Lambda t_{1}, \ldots, \Lambda t_{m_{i}}\right) \in \tilde{\phi}_{i, x}
$$

only if

$$
\left(b_{i \lambda}, x, t_{1}, \ldots, t_{m_{i}}\right) \in \tilde{\phi}_{i, x} .
$$

7. The algebra $\mathcal{O}$ of observation procedures is the algebra of realvalued continuous functionals $F$ on a linear carrier space $\Omega$. This space $\Omega$ is spanned by $N$ irreducible covariant-tensor-valued functions $\phi_{i}$ $(i=1, \ldots, N)$ on Minkowski space. Let

$$
\begin{gathered}
\phi_{i}: M \rightarrow T_{i} \quad\left[M \ni x \mapsto v_{i}(x)\right] \\
v_{i}(x): V^{\otimes m_{i}} \rightarrow R \\
{\left[V^{\otimes m_{\imath}} \ni\left(t_{1}, t_{2} \ldots t_{m_{i}}\right) \mapsto \phi_{i}\left(t_{1}, t_{2} \ldots t_{m_{i}}, x\right)\right] .}
\end{gathered}
$$

In other words, let the $\phi_{i}: M \rightarrow T_{i}$ be multilinear maps on the $m_{i}$-fold Cartesian product of vector spaces $V$ into the reals. An element $\Lambda$ of the Lorentz group

$$
\Lambda: V \rightarrow V
$$

induces a transformation of $T_{i}$ through $\left(t_{1}, t_{2}, \ldots, t_{m_{i}}\right) \mapsto\left(\Lambda t_{1}, \Lambda t_{2}, \ldots, \Lambda t_{m_{i}}\right)$. The functions $\phi_{i}$ are bounded and differentiable with respect to $x$. The 
covariant tensors form a linear vector space, and the set $T_{i}$ spans an irreducible subspace.

8. The images $F_{i, x, t_{1}, \ldots, t_{m_{i}}}$ of the field elements are linear functionals. Specifically,

$$
\begin{aligned}
\tilde{\phi}\left(b_{i \lambda}, x, t_{1}, \ldots, t_{m_{i}}\right)\left(\phi_{j}\right) \\
\quad=F_{i, x, t_{1}, \ldots, t_{m_{i}}}\left(\phi_{j}\right) \\
\quad=\delta_{i j} \phi_{j}\left(t_{1}, \ldots, t_{m_{\imath}}, x\right) .
\end{aligned}
$$

They will, for short, also be called fields.

9. Let $\left\{l^{\mu}\right\}(\mu=1, \ldots, 4)$ be an orthonormal basis in $V$, and

$$
\phi_{i}\left(l^{\mu_{1}}, l^{\mu_{2}}, \ldots, \mu^{\mu_{m_{i}}}, x\right)=\phi_{i, \mu_{1}, \mu_{2}, \ldots \mu_{m_{i}}}(x)
$$

be the tensor components in this basis. With a norm

$$
\left\|\phi_{i}\right\|=\operatorname{Sup}_{\substack{x \in M \\ l^{\mu} \in V}}\left(\left|\phi_{i, \mu_{1}, \ldots \mu_{m_{i}}}(x)\right|+\sum_{j=1}^{4}\left|\frac{\partial \phi_{i, \mu_{1}, \ldots, \mu_{m_{i}}}}{\partial x_{j}}\right|\right)
$$

where $\left|l^{\mu} l_{\mu}\right|=1$ and $\left\|\Sigma \lambda_{i} \phi_{i}\right\|=\left(\Sigma \lambda_{i}^{2}\left\|\phi_{i}\right\|^{2}\right)^{1 / 2}$, the linear space $\Omega$ is a Banach space. The space of functionals $F$ on $\Omega$ is equipped with the $c$-topology $[2,3]$. For a space-like hyperplane $h$, the subalgebra generated by the images $\tilde{\Phi} \tilde{\phi}_{i}(x)$ of the fields in $h$ is called the canonical subalgebra $\mathcal{O}_{h c}$ :

$$
\mathcal{O}_{c h}=\overline{\left\{F_{\left.i, x, t_{1}, \ldots t_{m_{i}}\right\}_{x \in h}}\right.},
$$

where the bar denotes topological closure.

10. The subset $\mathscr{S}_{h} \subset \mathscr{S}$ of state-preparing procedures associated to a space-like hyperplane $h$ is the set of states (i.e., linear, positive, continuous and normalized forms) on the subalgebra $\mathcal{O}_{c h}$.

The convex linear closure

$$
\mathscr{S}=\overline{\bigcup_{h \subset M} \mathscr{S}_{h}}
$$

of all these subsets $\mathscr{S}_{h}$ is the set $\mathscr{S}$.

11. To each expectation $\mathscr{E} \in\{\mathscr{E}\}$ there is associated a map $\Phi(\mathscr{E}): \mathcal{O} \rightarrow \mathscr{A}$ such that each subalgebra $\mathcal{O}_{c h} \subset \mathcal{O}$ is mapped isomorphically onto $\mathscr{A}$. This map induces a map $\Psi(\mathscr{E}): \mathscr{S} \rightarrow S(\mathscr{A})$ onto the set $S(\mathscr{A})$ of states on $\mathscr{A}$ by the requirement: $\mathscr{E}(s, \alpha)=\Psi_{S}(\Phi \alpha)$. All expectations with these properties are members of the collection $\{\mathscr{E}\}$.

The topology of $\mathscr{A}$ is that inherited from $\mathcal{O}$. The concrete realization of the algebra $\mathscr{A}$ of observables is the Abelian topological algebra of real-valued continuous functionals $f$ on the linear space $\omega$ spanned by covariant tensor-valued $C^{1}$ functions $\psi_{i}$ on Euclidean 3-space. The 
topology of $\omega$ is as defined in assumption 9 except for the substitution of 3-space for $M$.

12. A differentiable transformation $g: M \rightarrow M$ of Minkowski space induces a homeomorphism $h_{g}: \Omega \rightarrow \Omega$ through the definition

by

$$
(g t)_{\mu}=\left[\partial(g x)_{\mu} / \partial x_{v}\right] t_{v}
$$

$$
h_{g} \phi_{i, \lambda, t_{1}, \ldots, t_{m}}(x)=\phi_{i, \lambda, g t_{1}, \ldots, g t_{m}}(g x) \text {. }
$$

This homeomorphism $h_{g}$ induces, in turn, an automorphism $V_{g}: \mathcal{O} \rightarrow \mathcal{O}$ through

$$
V_{g} F(\phi)=F\left(h_{g} \phi\right) .
$$

The motion-induced (i.e., blueprint-preserving) permutations $\tilde{V}_{g}$ of $\tilde{\mathcal{O}}$ induce automorphisms of $\mathcal{O}$ through this chain.

\section{Equations of Motion}

Consider the canonical algebras $\mathcal{O}_{t c}$ associated to a family of parallel space-like hyperplanes with equations $t=$ const. By Section 10, Assumption 11 , each morphism $\Phi(\mathscr{E}): \mathcal{O} \rightarrow \mathscr{A}$ maps each subalgebra $\mathcal{O}_{t c}$ isomorphically onto $\mathscr{A}$. The automorphism $V_{t}: \mathcal{O} \rightarrow \mathcal{O}$ induced by the Poincaré translation $\left(x_{0}, \boldsymbol{x}\right) \rightarrow\left(x_{0}+t, \boldsymbol{x}\right)$ of Minkowski space induces an isomorphism $\mathcal{O}_{t c} \cong \mathcal{O}_{\tau+t, c}$ in any pair of this family $\left\{\mathcal{O}_{t c}\right\}$. Hence, the automorphism $V_{t}$, restricted to the family $\left\{\mathcal{O}_{t c}\right\}$, is compatible with the morphism $\Phi$ and induces an automorphism $Q_{t, \tau}$ of (Fig. 3), through

$$
\Phi V_{t} \alpha=Q_{t, \tau} \Phi \alpha \quad\left(\alpha \in \mathcal{O}_{\tau c}\right) .
$$

In general, the automorphisms $Q_{t, \tau}$ do not form a group. Let $\alpha$ be a field functional $F_{i, x, t_{1}, \ldots, t_{m_{i}}}$, where $x=(\tau, x)$. These functionals are differentiable, since by Assumption 1 of Section 5, the elements of the carrier space are differentiable.

By differentiating Eq. (11.1), we obtain

$$
\Phi \frac{\partial F}{\partial t}=\frac{\partial}{\partial t}\left(Q_{t, \tau}\right) \Phi F
$$

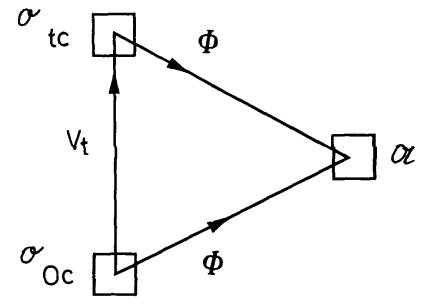

Fig. 3 
According to Theorem 4 of Ref. [4], the observable $\Phi V_{t} F$ is a member of the canonical algebra $\mathscr{A}_{c, V, \tau}$ associated to a sphere $V$ of radius $t$ and to the instant $\tau$. As $t$ goes to zero, this algebra shrinks to the point algebra $\mathscr{A}_{\tau, \boldsymbol{x}}$. With the notation

$$
\Phi F_{i, \boldsymbol{x}}=f_{i, \boldsymbol{x}},
$$

Equation (6.2) becomes

$$
\left(\frac{\partial f_{i}}{\partial t}\right)_{\tau, \boldsymbol{x}}=b_{i},
$$

where $b_{i} \in \mathscr{A}_{\tau, \boldsymbol{x}}$. Since $b_{i}$ belongs also to the canonical algebra associated to the hyperplane $t=\tau$, it can be expressed as a function of the field images $f_{i, \boldsymbol{x}}$ and of their space derivatives.

A Lorentz transformation $\Lambda$ with $(\tau, x)$ as fixed point carries the hyperplane $\tau=$ const into another hyperplane $\tau^{\prime}=$ const. This transformation $\Lambda$ induces a linear transformation of the field images $f_{i}(x)$ and of their partial derivatives. Since the transformed equation of motion is of first order in the new time derivative, the quantity $b_{i}$ can be expressed as a function of the field images $f_{i, x}$ and their first space derivatives, linear with respect to the latter:

$$
\left(\frac{\partial f_{i}}{\partial t}\right)_{x}-b_{i}\left(f_{i}, \frac{\partial f_{i}}{\partial x_{k}}\right)=0 \text {. }
$$

To summarize, the equations of motion are of first order in all directions and linear in the partial derivatives, but in general, nonlinear in the field images $f_{i, x}$. The equations of motion determine the automorphisms $Q_{t, \tau}$ of Eq. (11.1) and hence the expectation $\mathscr{E}$ that induces the $\operatorname{map} \Phi(\mathscr{E}): \mathscr{O} \rightarrow \mathscr{A}$.

\section{Equivalence Principle (First Part)}

The mathematical counterpart of the equivalence principle stated in operational terms [Eq. (6.1)] is

$$
\exists \mathscr{E}_{g} \in\{\mathscr{E}\} \mid \mathscr{E}(s, \alpha)=\mathscr{E}_{g}\left(s, V_{g} \alpha\right), \quad \alpha \in \mathcal{O}_{x} .
$$

By virtue of Assumption 11 of Section 10, the statement can be written as

$$
\exists \mathscr{E}_{g} \in\{\mathscr{E}\} \mid \Psi(\mathscr{E}) s[\Phi(\mathscr{E}) \alpha]=\Psi\left(\mathscr{E}_{g}\right) s\left[\Phi\left(\mathscr{E}_{g}\right) V_{g} \alpha\right], \quad\left(\alpha \in \mathcal{O}_{x}\right) .
$$

If this is applied to an element $\alpha \in \mathcal{O}_{x}$ for which $\Phi(\mathscr{E}) \alpha=0$, the equation states that all states $\left\{\Psi\left(\mathscr{E}_{g}\right) s\right\}$ vanish on the functional $\Phi\left(\mathscr{E}_{g}\right) V_{g} \alpha$. Since the states separate the algebra $\mathscr{A}$, this functional vanishes. We choose 
the natural inverse image in $\mathcal{O}$ of the expression (11.5):

$$
\Phi(\mathscr{E})\left[\frac{\partial F_{i}}{\partial t}-b_{i}\left(F_{i}, \frac{\partial F_{i}}{\partial x_{k}}\right)\right]=0 .
$$

Hence, the equation of motion associated to the altered expectation $\mathscr{E}_{g}$ is

$$
\Phi\left(\mathscr{E}_{g}\right) V_{g}\left[\frac{\partial F_{i}}{\partial t}-b_{i}\left(F_{i}, \frac{\partial F_{i}}{\partial x_{k}}\right)\right]=0
$$

We will show that there exists an expectation $\mathscr{E}_{g}$ that satisfies this equation. Since the expression in brackets is a member of the point algebra $\mathcal{O}_{x}$, we may choose $V_{g}$ to be a member of the acceleration group $\mathscr{M}_{x}$.

According to Section 7 of Ref. [4], the field images $F_{i}$ are acceleration invariant. Hence, the problem reduces to finding the effect of $V_{g}$ induced by the acceleration subgroups $A_{\alpha, n}$ on derivatives $\partial F / \partial x_{\lambda}$, where $F$ is a field image. For simplicity, we will derive the result for a vector field $F_{x, t}$, where $t$ is a unit four-vector $(|t|=1)$. We recall that for $\psi \in \Omega$, one has, for a canonical point functional at $x=0$, the relation

$$
F_{0, t}(\psi)=\psi(t, 0)=\psi^{\mu}(0) t_{\mu},
$$

where the $t_{\mu}$ are Cartesian components of $t$. We have

$$
\begin{aligned}
{\left[V_{g}\left(\partial / \partial x^{\lambda}\right) F_{x, t}\right]_{x=0}(\psi) } & =\left(\partial / \partial x^{\lambda}\right)\left[F_{x, t}\left(h_{g} \psi\right)\right]_{x=0} \\
& =\left[\left(\partial / \partial x^{\lambda}\right) F_{x, t}\right]_{x=0}\left(h_{g} \psi\right),
\end{aligned}
$$

where $h_{g}$ is the homeomorphism $\Omega \rightarrow \Omega$ that induces $V_{g}$. But according to Section 4,

$$
F_{x, t}\left(h_{g} \psi\right)=(g \psi)(x) t_{\mu}=\frac{\partial(g x)^{\mu}}{\partial x^{\nu}} \psi^{\nu}(x) t_{\mu}
$$

and therefore

$$
\frac{\partial}{\partial x^{\lambda}}\left[F_{x, t}\left(h_{g} \psi\right)\right]=\frac{\partial^{2}(g x)^{\mu}}{\partial x^{\lambda} \partial x^{\nu}} \psi^{\nu} t_{\mu}+\frac{\partial(g x)^{\mu}}{\partial x^{\nu}} \frac{\partial \psi^{\nu}}{\partial x^{\nu}} t_{\mu}
$$

According to Section 9, we are interested in the limit $|x| \rightarrow 0$ along the positive time axis. According to Ref. [4], we have for the acceleration transformations $g_{i, n}(n \neq 0)$

$$
\left[(g x)^{\mu} / x^{v}\right]_{x=0}=\delta_{\mu v} .
$$

Thus, we obtain

$$
\left[V_{g} \frac{\partial}{\partial x^{\lambda}} F_{x, t}\right]_{x=0}(\psi)=\left(\frac{\partial \psi^{\mu}}{\partial x^{\lambda}} t_{\mu}+\Delta_{v \lambda}^{\mu} \psi^{v} t_{\mu}\right)_{x=0},
$$


where

$$
\Delta_{v \lambda}^{\mu}=\lim _{|x| \rightarrow 0} \frac{\partial^{2}(g x)^{\mu}}{\partial x^{\lambda} \partial x^{\nu}}
$$

For later purposes, let us define

$$
\Gamma_{v \lambda}^{\varrho}=\frac{\partial^{2}(g x)^{\mu}}{\partial x^{v} \partial x^{\lambda}} \frac{\partial x^{\varrho}}{\partial(g x)^{\mu}}
$$

and observe that

$$
\lim _{|x| \rightarrow 0} \Gamma_{v \lambda}^{\varrho}=\Delta_{v \lambda}^{\varrho}
$$

Thus, the effect of the automorphism $V_{g}$ on the functional

$$
\frac{\partial F_{i}}{\partial t}-b_{i}\left(F_{i}, \frac{\partial F_{i}}{\partial x_{k}}\right)
$$

is to add the corrective term $\Gamma_{\nu \lambda}^{\varrho} \psi^{\nu}$ to the first derivative $\partial \psi^{\mu} / \partial x^{\lambda}$ wherever it occurs in the argument. Thus, we have shown the existence of an expectation $\mathscr{E}_{g}$ that satisfies Eq. (12.2). The equations of motion that determine it are to be obtained from those of $\mathscr{E}$ by the prescription: add the term $\Gamma_{\nu \lambda}^{Q} \psi^{v}$ to each partial derivative in the argument. Naturally, there exists a large family of such expectations $\mathscr{E}_{g}$, because the equation of motion is only determined at the point $x=0$.

Students of the conventional curved-space theory will perceive the similarity of our prescription with the usual practice of replacing ordinary by covariant derivatives. However, our space being flat, the similarity is purely formal at this point.

The values of $(g x)^{\mu}$ for a linear acceleration $(n=1)$ in the $x^{3}$ direction are, according to Ref. [4],

$$
\begin{aligned}
& (g x)^{1}=x^{1} \\
& (g x)^{2}=x^{2} \\
& (g x)^{3}=x^{3} \cosh \alpha|x|+x^{4} \sinh \alpha|x|, \\
& (g x)^{4}=x^{3} \sinh \alpha|x|+x^{4} \cosh \alpha|x| .
\end{aligned}
$$

After some calculation, one finds

$$
\begin{aligned}
& \Delta_{44}^{3}=-2 \alpha, \\
& \Delta_{44}^{4}=\Delta_{43}^{4}=-\alpha,
\end{aligned}
$$


and, in general, for a linear acceleration along $x_{i}$,

$$
\begin{aligned}
& \Delta_{44}^{i}=-2 \alpha, \\
& \Delta_{i 4}^{4}=\Delta_{4 i}^{4}=-\alpha .
\end{aligned}
$$

In the case of rotational acceleration, the definition of the acceleration group in the Ref. [4] gives

$$
\begin{aligned}
& (g x)^{1}=x^{1} \cos \theta|x|+x^{2} \sin \theta|x|, \\
& (g x)^{2}=-x^{1} \sin \theta|x|+x^{2} \cos \theta|x|, \\
& (g x)^{3}=x^{3}, \\
& (g x)^{4}=x^{4},
\end{aligned}
$$

and we find

$$
\Delta_{v \lambda}^{\mu}=0
$$

which, in some sense, reflects the point nature of our algebras $\mathcal{O}_{x}$. While a linear acceleration of a point observation procedure produces an effect, a rotational movement (whether accelerated or uniform) does not. Coriolis forces do not exist for a point algebra.

\section{Equivalence Principle (Second Part)}

It was shown in Section 12 that there exists an altered equation of motion (in loose language, a force) that produces the same effect as a bodily acceleration of measuring instruments. We wish to show that this "force" deserves the name "gravitational" in that its effect on the motion of otherwise free particles is independent of their mass - i.e., that it satisfies the "weak equivalence principle".

The theory of continuously distributed matter is appropriate to our field theoretical scheme. The equation of motion [11] of a matter field with invariant mass density $\mu_{0}$ is

$$
\mu_{0} \frac{\partial}{\partial x_{\mu}}\left(U_{\mu} U_{v}\right)=0
$$

The altered equation of motion (due to gravitational force in the usual language) is obtained from (13.1) by adding to the partial derivative a term that depends only on the acceleration and not on $\mu_{0}$. It is then clear 
that the flow lines of the mass field are altered by this addition in a manner independent of $\mu_{0}$. Hence, mass does not affect the motion of bodies in a gravitational field, in accordance with the weak equivalence principle.

\section{Measurements of Space-Time Distances}

It was assumed in Section 2 that observation instruments can be unambiguously located in Minkowski space by appropriately correcting for the bending of light rays. Consistency requires that the theory of observables developed on this basis should provide the means for this correction, so that observation instruments can be treated as objects of observation. This consistency requirement is substantially what Trautman [12] calls the Principle of Relativity: a theory should not contain "absolute elements" (in this case, straight lines) without also giving means for measuring them. It is in accordance with this principle that Einstein discarded the ether, because its uniform motion relative to an inertial observer was in principle unobservable.

Consider a particular class of states for which the electromagnetic energy is nonzero only near the origin $x=0$ and the state is vacuumlike elsewhere at $t=0$. Then, observations of the Poynting vector at various points will define the path of the light signal or signals.

The problem is now the converse: from the paths of light signals, to determine the locations of points in $M$. For this purpose, it is convenient to change the representation of the algebra $\mathscr{A}$. This will be done in two steps.

The generating functionals $f_{i, x}$ of $\mathscr{A}$ are carried by a time-translation automorphism $Q_{t}: \mathscr{A} \rightarrow \mathscr{A}$ into

$$
Q_{t} f_{i, x} \equiv f_{i, x^{\prime}} \quad(x=\boldsymbol{x}, t) .
$$

Local subalgebras $\mathscr{A}(R)$ are generated by the topological closure

$$
\bigcup_{x \in R} f_{i, x}=\mathscr{A}(R),
$$

if $R$ is an open set. The map $\Phi$ preserves the local structure in the sense that

$$
\Phi \mathcal{O}(R)=\mathscr{A}(R),
$$

but the map is, of course, not one-one: some elements $\alpha \notin \mathcal{O}(R)$ are mapped into $\mathscr{A}(R)$. When $\mathscr{A}$ is presented in this manner, as containing space-time subalgebras, there is a more natural carrier space for it than $\omega$.

Let

$$
Q_{\tau} f_{i,(\boldsymbol{x}, t)} \equiv f_{i,(\boldsymbol{x}, t+\tau)}
$$


and since the functionals $f_{i,(x, t)}$ generate $\mathscr{A}$, this transformation determines the automorphism. We define

$$
f_{i,(\boldsymbol{x}, t)}(\psi)=f_{i,(\boldsymbol{x}, 0)}\left(q_{t} \psi\right),
$$

and by Theorem (10.6) of Ref. [13] the homeomorphism $q_{t}$ of $\omega$ that induces the transformation

$$
f_{i, x} \rightarrow f_{i,(\boldsymbol{x}, t)}
$$

is unique. One can define functions $\chi$ on $M$ by

$$
\chi(x, t)=q_{t} \psi(x)
$$

The set of functions $\chi$ on $M$ will in general be a nonlinear set. The first change of representation consists in substituting the carrier space $\omega_{M}$ of functions $\chi$ on $M$. If $q_{t}$ is a group of homeomorphisms induced by a differential equation, then the space $\omega_{M}$ is that of the solutions of the differential equation. This nonlinear "space of solutions or orbits", is frequently used in classical mechanics.

Thr next step consists in identifying the three-index symbols $\Gamma_{\nu \lambda}^{\varrho}$ in Section 12 with affine connections in a pseudo-Riemannian manifold. More precisely, we consider a homeomorphism $h: M \rightarrow \mathscr{M}$ on Minkowski space onto a pseudo-Riemannian manifold such that the coefficients $\Gamma$ are the affine connections in Cartesian coordinates $x^{\mu}$. Then, the nonlinear space $\omega_{M}$ of functions $\chi$ on the linear space $M$ is replaced by a nonlinear space of functions $\chi_{\mathscr{M}}$ on the nonlinear manifold $\mathscr{M}$.

The solutions of Maxwell's equations with ordinary derivatives replaced by covariant derivatives as in Ref. [7] then have characteristics that are images of null geodesics in $\mathscr{M}$.

It was assumed in Section 1 that world lines of test particles were asymptotically straight. Hence, the pseudo-Riemannian manifold $\mathscr{M}$ is asymptotically flat, and all geodesics are asymptotically straight lines.

We now return to the problem of locating physical points in Minkowski space. The measurement procedures needed to find the curvature and affine connection of $\mathscr{M}$ can be reduced to the use of clocks and light signals. Having thus determined the affine connection and the location of a point in $\mathscr{M}$, there is a unique homeomorphism that agrees with observed straight world lines of distant objects. Of course, finding this homeomorphism requires global and not only local information, in accordance with what Trautman [12] calls equivalence principle.

The authors gratefully acknowledge stimulating discussions with P. Bergmann, R. Geroch, R. Haag, P. Havas, and M. A. Melvin. 


\title{
References
}

1. Ekstein, H.: Phys. Rev. 153, 1937 (1967)

2. Ekstein, H.: Phys. Rev. 184, 1315 (1969)

3. Avishai, Y., Ekstein, H., Moyal, J.: J. Mat. Phys. 13, 1139 (1972)

4. Avishai, Y., Ekstein, H.: Phys. Rev. D7, 983 (1973)

5. Wigner, E. P.: Rev. Mod. Phys. 29, 255 (1957)

6. Mackey,G.W.: Mathematical foundation of quantum mechanics. New York: Benjamin 1963

7. Havas, P.: Rev. Mod. Phys. 36, 938 (1964)

8. Bergmann,P.G.: In: Flugge, S. (Ed.): Encyclopedia of Physics, Vol. IV. Berlin: Springer 1962

9. Rickart, C.E.: General theory of Banach algebras. Princeton, N. J.: Van Nostrand 1960

10. Kastler,D.: Introduction à l'électrodynamique quantique, p. 50. Paris: Dunod 1961

11. Møller, C.: The theory of relativity. London: Oxford University Press 1952

12. Trautman, A.: In: General relativity. Englewood Cliffs. Prentice-Hall 1964

13. Gillman, L., Jerison, M.: Rings of continuous functions, p. 142. Princeton, N.J.: Van Nostrand 1960

Communicated by R. Haag

\author{
H. Ekstein \\ Université Aix-Marseille II \\ Centre de Luminy \\ Département de Physique \\ Section Théorique \\ 70, Route Léon Lachamp \\ F-70 Marseille $\left(9^{\mathrm{e}}\right)$, France
}

